\title{
Concern and Usefulness of Intratumoral Injection of Ethyl Alcohol for Devascularization of Intracranial Tumors
}

\author{
Guru Dutta SATYARTHEE ${ }^{1}$, Sushil KUMAR² \\ ${ }^{1}$ All India Institute of Medical Sciences New Delhi, Department of Neurosurgery, Delhi, India \\ ${ }^{2}$ St Stephens Hospital Delhi, Department of Neurosurgery, Delhi, India
}

To the Editor;

$\mathrm{W}$ Te read with interest the article "Intratumoral ethyl alcohol injection for devascularization of hypervascular intracranial tumors" (3). Every neurosurgeon is very well aware that excessive intraoperative bleeding can create havoc in the operating theatre. Preoperative embolization of an intracranial tumor can lead to significant reduction in vascularity as commonly observed during surgery. However, a few patients may not be suitable for such preoperative embolization procedures due to tortuous feeding vessels, unavailability of an endovascular facility, inability to perform preoperative embolization, impossibility of emergency surgical intervention, and few cases may develop intraoperative severe bleeding despite embolization.

Tumturk et al. reported an additional potent hemostatic procedure in the neurosurgical armamentarium, aiming to reduce vascularity of tumor with the use of intratumoral absolute alcohol injection with an excellent result (3). Ethyl alcohol is inexpensive and easily available. Tumturk et al. meticulously described the method of intratumoral injection under neuronavigation guidance, and reported their observation on a total of 55 cases with a hypervascular intracranial tumor. Intratumoral ethyl alcohol injection led to marked reduction in vascularity with relatively less bleeding and safer resection of tumor. The authors concluded that intratumoral ethyl alcohol injection is an easy, cheap and less invasive way to obtain effective and safe reduction of vascularity and it may constitute an alternative method in the event of intra-operative severe bleeding despite embolization. However, no serious complication was observed in the study (3).
The mechanism of action of intratumoral ethyl alcohol injection is dehydration and subsequent necrosis, denaturing the structure of the cellular proteins, resulting in complete ablation of the tumor. Pure alcohol also blocks blood flow to the tumor bed resulting in small blood vessel thrombosis that leads to tumor necrosis. Ethyl alcohol injection is also used for the treatment of liver and kidney tumors and vascular malformations (1).

The use of ethyl alcohol in neurosurgical cases is not new. In 1991, Xie et al. reported use of ultrasonography-guided percutaneous alcohol injection in cases suffering with recurrent intracerebral supratentorial gliomas (4). In 1998, Lonser et al. reported tumor devascularization by intratumoral ethanol injection during surgery in 3 spinal epidural metastases and one large cerebellar neoplasm (hemangioblastoma). They observed immediate blanching, devascularization and enhanced visualization. Incremental tumor devascularization was achieved by careful injection of small amounts of ethyl alcohol directly into the lesion, producing immediate and complete regional tumor devascularization (2).

However, there are many concerns on this technique. Firstly, an experienced anaesthetic team with facility of hemodynamic monitoring is required in order to help the patient for early detection and management of cardiovascular instability developing during the intratumoral alcohol injection. Second, a cost-benefit analysis has never been carried out regarding intratumoral ethyl alcohol injection. Nevertheless, the current technique is really a major advancement in field of controlling excessive bleeding during surgery, and using ethyl alcohol injection with meticulously followed precautions will be helpful in saving not only lives but also ensuring a good neurological outcome. 


\section{REFERENCES}

1 Kotoh K, Sakai H, Sakamoto S: The effect of percutaneous ethanol injection therapy on small solitary hepatocellular carcinoma is comparable to that of hepatectomy. Am J Gastroenterol 89: 194-198, 1994

2. Lonser RR, Heiss JD, Oldfield EH: Tumor devascularization by intratumoral ethanol injection during surgery. Technical note. J Neurosurg 88(5): 923-924, 1998
3. Tumturk A, Kucuk A, Canoz O, Tucer B, Kurtsoy A: Intratumoral ethyl alcohol injection for devascularization of hypervascular intracranial tumors. Turk Neurosurg 26(5): 684-689, 2016

4. Xie XL: Alcohol injections for the treatment of recurrent intracerebral gliomas. Zhonghua Wai Ke Za Zhi. 29(9): 571572, 1991 (In Chinese) 